\title{
Fuzzy Set Theoretic Approach to Collocation Extraction
}

\author{
Raj Kishor Bisht \\ Dept. of Computer Science \\ Amrapali Institute of Management \\ and Computer Application, \\ Haldwani (Uttarakhand)-India
}

\author{
H. S. Dhami \\ Dept. of Mathematics \\ University of Kumaun, \\ S.S.J.Campus Almora \\ (Uttarakhand) -India
}

\begin{abstract}
Fuzzy approach deals with the linguistic properties of elements such as beauty, coldness, hotness etc. Collocations are linguistically motivated. Decision of word combination for being collocation is a linguistic term as merely cooccurrence of word combinations does not signify the presence of collocation. Thus collocation extraction can be made possible by looking its linguistic aspect. In the present paper, an attempt has been made to make two different fuzzy sets of word combinations to be considered for collocations. Mutual information and t-test have been taken as basis for the construction of fuzzy sets. Two fuzzy set theoretical models have been proposed to identify collocations. It has been shown that fuzzy set theoretical approach works very well for collocation extraction. The working data has been based on a corpus of about one million words contained in different novels constituting project Gutenberg available on www.gutenberg.org.
\end{abstract}

\section{General Terms}

Natural Language processing, Computational lexicography

\section{Keywords}

Collocation, Fuzzy set, Mutual Information, t-test

\section{INTRODUCTION}

'Collocations' are a class of word groups which lie between idioms and free word combinations. However, it is typical to draw a line between a phrase and a collocation. Idioms and phrases may be defined as an expression in the language that is peculiar to itself. It becomes well nigh impossible to guess the meaning of an idiom from the word it contains (e.g. At the eleventh hour). And, moreover, the meanings that idioms have are often stronger than the meanings of non-idiomatic phrases. For instance, 'look daggers at someone' is more emphatic than 'look angrily at someone', although both of them have the same meaning [5]. On the other hand, in a free word combination, a word can be replaced by another word without seriously modifying the overall meaning of the composite unit so that one can not easily predict it from the remaining ones. For example, 'end of the day' can not be predicted from 'end of the lecture', if we replace 'day' by 'lecture'. According to Kathleen R. McKeown and Dragomir R. Radev [8] 'collocations are arbitrary, language specific, recurrent in context and common in technical language'. Collocations are utilized for many natural language applications such as, machine translation, computational lexicography, information retrieval, natural language generation etc [10]. Collocation translation improves the quality of machine translation. For example, 'public opinion' in English is 'janata ki raay' in Hindi, 'pocket money' in English is 'jeb kharch' in Hindi. Automatic identification of important collocations to be listed in a dictionary is the task of computational lexicography. Adequate knowledge of collocations can improve the performance of information retrieval system.

Statistical methods have shown a remarkable presence in collocation extraction. Frequency measure was used by Choueka et al [2] to identify a particular type of collocations. Church and Hanks [3] used mutual information to extract word pairs that tend to co-occur within a fixed size window (normally 5 words), in which extracted words may not be directly related. Smadja [11] extracted collocations through a multi-stage-process taking the relative positions of co-occurring words into account. Church and Gale [4] used the $\chi^{2}$ - test for the identification of translation pairs in aligned corpora. Collocations extraction and their use in finding word similarity was suggested by Dekang Lin [9]. The use of t-test to find words whose co-occurrence patterns best distinguish between two words was suggested by Church and Hanks [3]. Dunning [6] applied likelihood ratio test to collocation discovery. Marc Weeber et al [16] devised an extraction system for the full word frequency ranges which computes the significance of association by the log-likelihood ratio and Fisher's exact test. Diana Zaiu Inkpen and Graeme Hirst [15] extended a lexical knowledgebase of near-synonym differences with knowledge about their collocational behaviour. Pavel Pecina [13] made an empirical evaluation of a comprehensive list of automatic collocation extraction methods using precision-recall measures. Violeta Seretan and Eric Wehrli [14] pointed out several language-specific issues related to extraction and proposed a strategy for coping with them. Afsaneh Fazly and Suzanne Stevenson [12] identified several classes of multiword expressions.

Almost all the techniques of collocation extraction look at whether the probability of seeing a combination differs significantly from what we would expect from their component words and reject those word combinations that do not. To decide whether a word combination makes a collocation or not is a vague measurement. One can not apply a particular rule of collocation extraction for every word combinations; thus fuzzy approach is quite useful for collocation extraction. In a classical or crisp set we assign only two values 0 or 1 to different elements depend on their belongingness to the set. If an element is a member of the set then it is 1 otherwise 0 . This approach is well 
defined for exact properties, such as for the set of positive real numbers on a set of real numbers, we may assign 1 for every positive real number and 0 for every non positive real number but this approach does not work well for linguistic terms such as good student, hot temperature etc. because no well defined definition is there for such terms. Instead of assigning 0 or 1 , we use the closed interval $[0,1]$. For a fuzzy set, we define a grade of membership for each element which shows its degree of belongingness to the set. Zero grade of membership indicates that the element does not belong to the set and one grade of membership gives full support to the element for its belongingness to the set. We can assume a set of collocations in which every word combination is a member of the set with different grades of membership. In the present paper we have made an attempt to find out the membership function for word combinations by utilizing two previous approaches of collocation extraction, that is, mutual information and t-score. The working data has been based on 1 million words corpus compiled by taking some of the novels contained in project Gutenberg available at www.gutenberg.org/etext/<no.> (See appendix).

The structure of the paper is as follows: In Section 2, we have defined two fuzzy sets obtained by fuzzyfying the mutual information scores and t-scores. In section 3, fuzzy set theoretical model for collocation extraction has been proposed. Evaluation of the proposed model will be the part of section 4. Finally, Section 5 deals with the discussion and conclusions on the present study.

\section{FUZZYFICATION OF COLLOCATION EXTRACTION TECHNIQUES}

In this section, we have mentioned the two techniques of collocation extraction, that is, mutual information and t-score. We have found the grade of membership for each word combination based on these two methods.

\subsection{Mutual Information}

Mutual information from information theory has been utilized to find the closeness between word pairs by Church \& Hanks [3]. Mutual information for two events $\mathrm{x}$ and $\mathrm{y}$ is defined as:

$$
I(x, y)=\log _{2} \frac{P(x, y)}{P(x) \cdot P(y)}
$$

If we write $w_{1}$ and $w_{2}$ for the first and second word respectively, instead of $\mathrm{x}$ and $\mathrm{y}$, then the mutual information for the two words $w_{1}$ and $w_{2}$ is given by:

$$
I\left(w_{1}, w_{2}\right)=\log _{2} \frac{P\left(w_{1}, w_{2}\right)}{P\left(w_{1}\right) \cdot P\left(w_{2}\right)}
$$

where $P\left(w_{1}, w_{2}\right)$ is the probability of two words $w_{1}$ and $w_{2}$ coming together in a certain text and $P\left(w_{1}\right)$ and $P\left(w_{2}\right)$ are the probabilities of $w_{1}$ and $w_{2}$ appearing separately in the text, respectively.

If $P\left(w_{1}, w_{2}\right)=P\left(w_{1}\right) \cdot P\left(w_{2}\right)$, that is, the two words are independent to each other, then $I\left(w_{1}, w_{2}\right)=0$, which indicates that these two words are not good candidates for collocation. A high mutual information score signifies the presence of a collocation.

\subsubsection{Fuzzification of Mutual Information}

The term 'high mutual information score' is quite vague as it does not provide a basis to say which mutual information score can be considered as high. Let us consider a fuzzy set A of collocations, then each word combination will be a member of the set A with a particular grade of membership. Grade of membership can be defined with the help of mutual information scores. To find the grade of membership using the mutual information scores, we have analyzed the pattern of mutual information scores. Instead of looking for every mutual information score, we have classified mutual information scores into small class intervals and assigned ranks to word combinations according to the classes. So that word combinations falling under a class have same grade of membership. Table 1 shows the mutual information and their corresponding ranks.

Table 1: Mutual information scores and corresponding ranks.

$$
\text { Mutual Information score Rank }
$$

\begin{tabular}{cc}
\hline $0-0.25$ & 1 \\
$0.26-0.50$ & 2 \\
$0.51-0.75$ & 3 \\
$0.76-1.00$ & 4 \\
$\ldots \ldots \ldots \ldots \ldots \ldots \ldots \ldots \ldots \ldots$ & $\ldots$ \\
$\ldots \ldots \ldots \ldots \ldots \ldots \ldots$ & $\ldots$ \\
$12.76-13.00$ & 51 \\
$\ldots \ldots \ldots \ldots \ldots \ldots$
\end{tabular}

Class intervals have been taken too small as we have found that small class interval leads to accuracy in the prediction of ranks from the distribution. We can define a function to get the rank of a word combination from its mutual information. Let $x \in R^{+}$be the mutual information of a word combination and $y \in I^{+}$be the corresponding rank, then $f: R^{+} \rightarrow I^{+}$such that $y=f(x)=\left\lceil\frac{x}{0.25}\right\rceil .\lceil x\rceil$ is the ceiling function. 
To find the grade of membership from the rank, we can define an another function $g: I^{+} \rightarrow[0,1]$ such that $y=g(x)$, where $x \in I^{+}$is the rank and $y \in[0,1]$ is the corresponding grade of membership. For defining the function $g(x)$, we know that it should tend to zero when $x$ tends to one (lowest mutual information rank) and one when $x$ tends to infinity (highest mutual information score) respectively. Therefore we can define $g(x)$ as follows:

$$
y=g(x)=\frac{\log x}{\log (x+10)}
$$

From the function $y=g(x)$, it is clear that when $x \rightarrow 1, y \rightarrow 0$ and $x \rightarrow \infty, y \rightarrow 1$.

Finally grade of membership for a word combination based on the mutual information scores can be given as:

$$
A_{I}=g \text { of }(x)=g(f(x))
$$

We have taken the example of eighty word pairs from the compiled corpus. Table 2 shows the mutual information scores and their corresponding grades of membership for different word combinations.

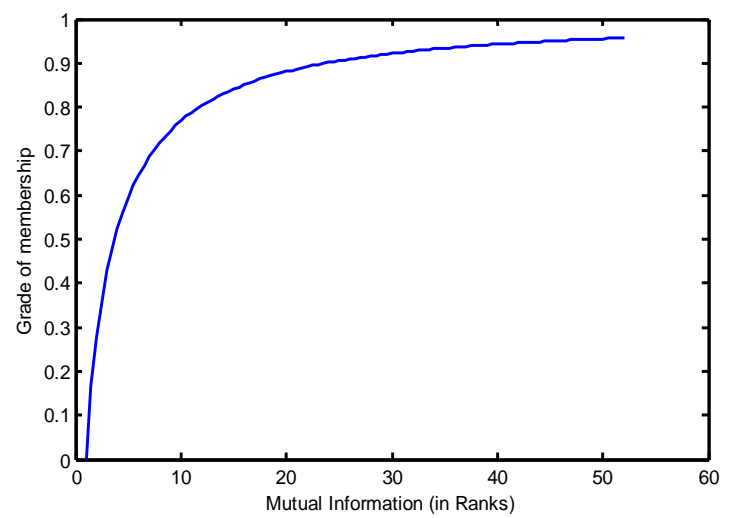

Fig.1: Grade of membership for mutual information scores

\subsection{The t-test}

The t-test has been used by Church \& Hanks [3] for collocation discovery to test the validity of a hypothesis. For that purpose, we formulate a null hypothesis $H_{0}$ that the two words $w_{1}$ and $w_{2}$ appear independently in the text. So under the null hypothesis $H_{0}$, the probability that the words $w_{1}$ and $w_{2}$ are coming together is simply given by:

$$
P\left(w_{1}, w_{2}\right)=P\left(w_{1}\right) \cdot P\left(w_{2}\right) .
$$

The null hypothesis has been tested by using t-test. If the null hypothesis is accepted, we conclude that the occurrence of two words is independent of each other. Otherwise, we may conclude that they depend on each other, that is, they form collocations. In t-test we use the null hypothesis that the sample is drawn from a distribution with mean $\mu$, taking sample mean and variance into account. The t-test considers the difference between the observed and expected mean. The $\mathrm{t}$ statistic is defined as:

$$
t=\frac{\bar{x}-\mu}{\sqrt{s^{2} / N}} \sim t_{n-1}(\alpha)
$$

where $\bar{x}$ is the sample mean, $s^{2}$ is the sample variance, $N$ is the sample size, $\mu$ is the mean of the distribution and $t_{n-1}(\alpha)$ denotes a t- distribution with (n-1) degrees of freedom at $\alpha$ level of significance. To apply t- test for testing the independence of two words $w_{1}$ and $w_{2}$, we assume that $f\left(w_{1}\right), f\left(w_{2}\right)$ and $f\left(w_{1}, w_{2}\right)$ are the respective frequencies of the word $w_{1}, w_{2}$ and $w_{1} w_{2}$ in the corpus and $N$ is the total number of words / bigrams in the corpus. Then, we have,

$$
\begin{aligned}
& P\left(w_{1}\right)=\frac{f\left(w_{1}\right)}{N} \quad\left(\text { say } p_{1}\right), P\left(w_{2}\right)=\frac{f\left(w_{2}\right)}{N} \quad\left(\text { say } p_{2}\right) \\
& P\left(w_{1}, w_{2}\right)=\frac{f\left(w_{1}, w_{2}\right)}{N} \quad\left(\text { say } p_{12}\right)
\end{aligned}
$$

The null hypothesis is

$$
H_{0}: P\left(w_{1}, w_{2}\right)=\boldsymbol{P}\left(w_{1}\right) \cdot \boldsymbol{P}\left(w_{2}\right)=p_{1} \cdot p_{2}
$$

If we select bigrams (word pairs) randomly then the process of randomly generating bigrams of words and assigning 1 to the outcome that the particular word combination for which we are looking for is a collocation and 0 to any other outcome follows a Bernoulli distribution. For the Bernoulli distribution we have

Mean $(\mu)=p$ and Variance $\left(\sigma^{2}\right)=p(1-p)$.

Thus, if the null hypothesis is true, the mean of the distribution is $\mu=p_{1} \cdot p_{2}$. Also, for the sample, we have $P\left(w_{1}, w_{2}\right)=p_{12}$. Therefore, using Binomial distribution, sample mean $\bar{x}=p_{12}$ and sample variance $s^{2}=p_{12}\left(1-p_{12}\right)$. Using (5), we calculate the value of $|t|$ and compare it with the tabulated value at given level of significance. If the value of $|t|$ for a particular bigram is greater than the value obtained from the table, we reject the null hypothesis, which indicates that the bigram may be 
considered as a collocation. We have chosen the level of significance $\alpha=.005$ for which $t=2.57$.

\subsubsection{Fuzzification of $t$-score}

To accept only those bigrams for collocations which have $|t|$ score greater than 2.57 is accurate as far as we take the $t$-test into consideration but extraction of collocation is not a pure mathematical job since the decision as to what constitutes a collocation is affected by its linguistic aspect also. This provides us a reason to think about those word combinations whose $t$ scores are less than 2.57 but very close to it. Therefore it opens a way to make a fuzzy set for collocations based on $t$ scores. The membership function for a word combination $\mathrm{x}$ can be defined as follows:

$$
A_{t}(x)= \begin{cases}1 & \text { if } \quad|t| \geq 2.57 \\ \frac{|t|}{2.57} & \text { otherwise }\end{cases}
$$

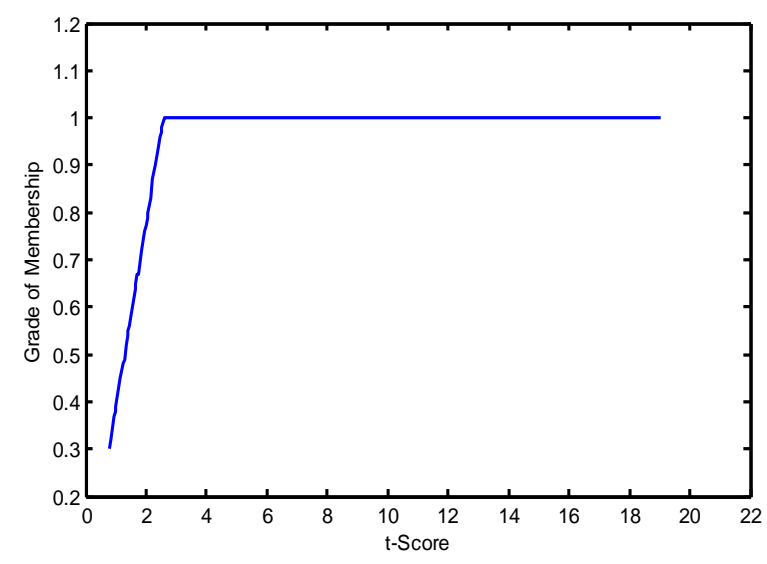

Fig.2: Grade of membership for collocation using t-score

\section{FUZZY DECISION MAKING FOR COLLOCATION EXTRACTION}

In this section, we have presented fuzzy set theoretical models for collocation extraction. In this model different opinions have been aggregated to find the fuzzy set of collocation. We have used the most common method based on the probabilistic interpretation of membership functions given by multiple experts [7]. Different experts are asked to for some $x \in X$ to valuate its belongingness to $A$, where $A$ is a fuzzy set on $X$ that represent a linguistic term associated with a given linguistic variable. For some $x \in X$,

let $A_{r}(x)$ denote the answer of the $r^{\text {th }}$ expert $(r \in N)$ in the term of belongingness of $x$ to $A$, where $A_{r}(x)$ has only two values 0 and 1 for $x \in A$ and $x \notin A$ respectively. Then the membership function can be defined as follows:

$$
A(x)=\frac{\sum_{r=1}^{n} A_{r}(x)}{n}, \text { where } \mathrm{n} \text { is the number of experts. }
$$

Here, for a word combination $x, A_{I}(x)$ and $A_{t}(x)$ be the opinions of the mutual information score and $\mathrm{t}$ - score respectively, in terms of grade of membership of $x$ to its belongingness to $A$. For a word combination $x$, The grade of membership can be given as $\quad A(x)=\frac{A_{I}(x)+A_{t}(x)}{2}$.

On using the above fuzzy decision model, we can obtain the grade of membership of each word combination for being collocation. A word combination that attains a maximum grade of membership can be taken as collocation.

\section{EVALUATION OF FUZZY SET THEORETIC APPROACH}

For evaluating the fuzzy set theoretic approach, we have taken the example of eighty word pairs from the compiled corpus. We have calculated the mutual information scores and t-scores for different word combinations and also their corresponding grades of membership. Table 2 shows the frequencies $f\left(w_{1}\right), f\left(w_{2}\right)$,

$f\left(w_{1} w_{2}\right)$ of words $w_{1}, w_{2}$ and their combination $w_{1} w_{2}$ respectively with their respective mutual information score, $\mathrm{t}$-score and grades of membership. Table 2 shows the grades of membership for different bigrams in the text using the fuzzy set theoretic model. We can choose different standards (grades of membership) for collocations extraction as values near to 1 show a high grade of membership for collocation. Validity of the word pairs given in table 2 have been checked through www.thefreedictionary.com, Cambridge Advanced learner's Dictionary and English to Hindi Translation point of view. Only 17 word pairs (star marked in table 2) have been found meaningful as collocations.

If we look at the translation of the some of the word pairs from English to Hindi we found that the words marked as asterisk in table 2 form meaningful combination. "Christmas eve" is translated as "Christmas ki purv sandhya", "Public opinion' is translated as "Janta ki raay" or "janmat", "human being" as "manav" or "manushya" , "young man" as "Naujawan" , "human nature" as "Manav Swabhaw" , "take care" as "kyayal rakhana".

On the basis of this, we can compare the results of mutual information and t-score with the results obtained by the proposed model. The mutual information does not provide a criterion for collocation extraction except saying high mutual information score shows the presence of a collocation. Precision and recall will depend upon the choice of the high mutual information score. However we can take different criteria of mutual information for calculating precision and recall. Table 3 shows the precision and recall for different mutual information scores. For t-score precision is $46 \%$ and recall is $70 \%$. Table 4 shows the precision and recall of the proposed models. 
Table 2: Mutual Information, t-score and the respective grades of membership for different word combinations.

\begin{tabular}{|c|c|c|c|c|c|c|c|c|c|}
\hline$W_{1}$ & $W_{2}$ & $f\left(w_{1}\right)$ & $f\left(w_{2}\right)$ & $f\left(w_{1} w_{2}\right)$ & $M I$ & $A_{i}$ & $|t|$ & $A_{t}$ & $A$ \\
\hline${ }^{*}$ Christmas & eve & 72 & 33 & 9 & 11.96 & 0.95 & 3.00 & 1.00 & 0.98 \\
\hline *base & camp & 54 & 55 & 7 & 11.27 & 0.95 & 2.64 & 1.00 & 0.98 \\
\hline *public & opinion & 190 & 103 & 10 & 9.07 & 0.94 & 3.16 & 1.00 & 0.97 \\
\hline *both & sides & 409 & 69 & 11 & 8.68 & 0.93 & 3.31 & 1.00 & 0.97 \\
\hline *human & being & 182 & 735 & 30 & 7.88 & 0.93 & 5.45 & 1.00 & 0.97 \\
\hline *great & deal & 911 & 118 & 20 & 7.61 & 0.92 & 4.45 & 1.00 & 0.96 \\
\hline *human & nature & 182 & 251 & 7 & 7.33 & 0.92 & 2.63 & 1.00 & 0.96 \\
\hline strong & enough & 172 & 657 & 13 & 6.92 & 0.91 & 3.58 & 1.00 & 0.96 \\
\hline *more & than & 2124 & 1563 & 369 & 6.87 & 0.91 & 19.05 & 1.00 & 0.96 \\
\hline *take & care & 808 & 228 & 20 & 6.83 & 0.91 & 4.43 & 1.00 & 0.96 \\
\hline *young & man & 741 & 2138 & 147 & 6.61 & 0.91 & 12.00 & 1.00 & 0.96 \\
\hline *early & days & 182 & 497 & 7 & 6.35 & 0.91 & 2.61 & 1.00 & 0.96 \\
\hline long & journey & 967 & 100 & 7 & 6.25 & 0.91 & 2.61 & 1.00 & 0.96 \\
\hline last & night & 846 & 856 & 47 & 6.09 & 0.90 & 6.76 & 1.00 & 0.95 \\
\hline${ }^{*}$ fire & bucket & 291 & 15 & 5 & 10.23 & 0.94 & $2.23^{\star *}$ & 0.87 & 0.91 \\
\hline away & from & 862 & 3945 & 135 & 5.38 & 0.89 & 11.34 & 1.00 & 0.95 \\
\hline came & along & 1360 & 367 & 13 & 4.77 & 0.88 & 3.47 & 1.00 & 0.94 \\
\hline every & night & 676 & 856 & 13 & 4.56 & 0.87 & 3.45 & 1.00 & 0.94 \\
\hline trench & life & 99 & 1102 & 6 & 5.85 & 0.90 & $2.41^{* *}$ & 0.94 & 0.92 \\
\hline night & before & 856 & 1164 & 18 & 4.25 & 0.86 & 4.02 & 1.00 & 0.93 \\
\hline must & take & 1144 & 808 & 16 & 4.18 & 0.86 & 3.78 & 1.00 & 0.93 \\
\hline last & time & 846 & 1463 & 20 & 4.09 & 0.85 & 4.21 & 1.00 & 0.93 \\
\hline strong & man & 172 & 2183 & 7 & 4.29 & 0.86 & $2.51^{* *}$ & 0.98 & 0.92 \\
\hline long & after & 967 & 1304 & 18 & 3.91 & 0.85 & 3.96 & 1.00 & 0.93 \\
\hline might & even & 1143 & 768 & 10 & 3.58 & 0.83 & 2.90 & 1.00 & 0.92 \\
\hline come & over & 1358 & 1394 & 19 & 3.40 & 0.83 & 3.95 & 1.00 & 0.92 \\
\hline${ }^{*}$ look & upon & 756 & 1913 & 12 & 3.12 & 0.81 & 3.07 & 1.00 & 0.91 \\
\hline little & episode & 1630 & 16 & 4 & 7.33 & 0.92 & $1.99^{* *}$ & 0.77 & 0.85 \\
\hline almost & every & 518 & 676 & 6 & 4.17 & 0.86 & $2.31^{* *}$ & 0.90 & 0.88 \\
\hline *evil & eye & 124 & 259 & 4 & 7.03 & 0.92 & $1.98^{\star *}$ & 0.77 & 0.85 \\
\hline time & before & 1463 & 1164 & 11 & 2.76 & 0.79 & 2.83 & 1.00 & 0.90 \\
\hline long & way & 967 & 1084 & 8 & 3.00 & 0.80 & $2.48^{\star}$ & 0.96 & 0.88 \\
\hline painful & experience & 27 & 87 & 3 & 10.39 & 0.95 & $1.73^{* *}$ & 0.67 & 0.81 \\
\hline make & use & 963 & 222 & 5 & 4.62 & 0.87 & $2.15^{\star \star}$ & 0.83 & 0.85 \\
\hline very & like & 1410 & 1602 & 11 & 2.36 & 0.76 & 2.67 & 1.00 & 0.88 \\
\hline dark & shadow & 279 & 93 & 3 & 6.92 & 0.92 & $1.72^{\star \star}$ & 0.67 & 0.80 \\
\hline last & century & 846 & 32 & 3 & 6.86 & 0.91 & $1.72^{* *}$ & 0.67 & 0.79 \\
\hline like & some & 1602 & 1786 & 11 & 2.01 & 0.72 & $2.50^{* *}$ & 0.97 & 0.85 \\
\hline cheerful & noise & 48 & 720 & 3 & 6.51 & 0.91 & $1.71^{* *}$ & 0.67 & 0.79 \\
\hline night & air & 856 & 475 & 5 & 3.69 & 0.84 & $2.06^{* *}$ & 0.80 & 0.82 \\
\hline
\end{tabular}




\begin{tabular}{|c|c|c|c|c|c|c|c|c|c|}
\hline$W_{1}$ & $W_{2}$ & $f\left(w_{1}\right)$ & $f\left(w_{2}\right)$ & $f\left(w_{1} w_{2}\right)$ & $M I$ & $A_{i}$ & $|t|$ & $A_{t}$ & $A$ \\
\hline only & because & 1187 & 371 & 5 & 3.58 & 0.83 & $2.05^{\star *}$ & 0.80 & 0.82 \\
\hline your & book & 2888 & 153 & 5 & 3.57 & 0.83 & $2.05^{\star *}$ & 0.80 & 0.82 \\
\hline another & half & 693 & 696 & 5 & 3.45 & 0.83 & $2.03^{* *}$ & 0.79 & 0.81 \\
\hline welcome & relief & 70 & 71 & 2 & 8.72 & 0.93 & $1.41^{* *}$ & 0.55 & 0.74 \\
\hline *national & guard & 39 & 163 & 2 & 8.37 & 0.93 & $1.41^{* *}$ & 0.55 & 0.74 \\
\hline good & terms & 1299 & 88 & 3 & 4.79 & 0.88 & $1.67^{\star *}$ & 0.65 & 0.77 \\
\hline horrible & thing & 55 & 580 & 2 & 6.04 & 0.90 & $1.39^{* *}$ & 0.54 & 0.72 \\
\hline stark & madness & 6 & 22 & 1 & 12.96 & 0.96 & $1.00^{* *}$ & 0.39 & 0.68 \\
\hline usual & hour & 115 & 344 & 2 & 5.73 & 0.90 & $1.39^{* *}$ & 0.54 & 0.72 \\
\hline step & towards & 135 & 348 & 2 & 5.48 & 0.89 & $1.38^{* *}$ & 0.54 & 0.72 \\
\hline away & down & 862 & 1517 & 6 & 2.27 & 0.75 & $1.94^{* *}$ & 0.76 & 0.76 \\
\hline valid & reason & 6 & 151 & 1 & 10.18 & 0.94 & $1.00^{* *}$ & 0.39 & 0.67 \\
\hline might & still & 1143 & 799 & 5 & 2.52 & 0.77 & $1.85^{\star *}$ & 0.72 & 0.75 \\
\hline spiritual & creature & 15 & 62 & 1 & 10.14 & 0.94 & $1.00^{* *}$ & 0.39 & 0.67 \\
\hline rapid & motion & 32 & 42 & 1 & 9.61 & 0.94 & $1.00^{* *}$ & 0.39 & 0.67 \\
\hline clumsy & fashion & 11 & 126 & 1 & 9.57 & 0.94 & $1.00^{* *}$ & 0.39 & 0.67 \\
\hline like & myself & 1602 & 372 & 4 & 2.82 & 0.79 & $1.72^{* *}$ & 0.67 & 0.73 \\
\hline visible & effort & 27 & 73 & 1 & 9.06 & 0.94 & $1.00^{* *}$ & 0.39 & 0.67 \\
\hline empty & tent & 128 & 16 & 1 & 9.00 & 0.94 & $1.00^{* *}$ & 0.39 & 0.67 \\
\hline huge & space & 35 & 64 & 1 & 8.87 & 0.93 & $1.00^{* *}$ & 0.39 & 0.66 \\
\hline peasant & girl & 10 & 253 & 1 & 8.70 & 0.93 & $1.00^{* *}$ & 0.39 & 0.66 \\
\hline wild & dreams & 136 & 46 & 1 & 7.39 & 0.92 & $0.99^{* *}$ & 0.39 & 0.66 \\
\hline except & myself & 81 & 1602 & 2 & 4.02 & 0.85 & $1.33^{* *}$ & 0.52 & 0.69 \\
\hline wrong & way & 121 & 1084 & 2 & 4.00 & 0.85 & $1.33^{* *}$ & 0.52 & 0.69 \\
\hline round & upon & 387 & 1913 & 4 & 2.51 & 0.77 & $1.65^{\star *}$ & 0.64 & 0.71 \\
\hline last & link & 864 & 15 & 1 & 6.34 & 0.91 & $0.99^{* *}$ & 0.38 & 0.65 \\
\hline looking & through & 431 & 1010 & 3 & 2.86 & 0.79 & $1.49^{* *}$ & 0.58 & 0.69 \\
\hline human & affairs & 182 & 86 & 1 & 6.07 & 0.90 & $0.99^{* *}$ & 0.38 & 0.64 \\
\hline *water & level & 286 & 65 & 1 & 5.82 & 0.90 & $0.98^{* *}$ & 0.38 & 0.64 \\
\hline most & powerful & 723 & 33 & 1 & 5.46 & 0.89 & $0.98^{* *}$ & 0.38 & 0.64 \\
\hline along & over & 367 & 1394 & 3 & 2.62 & 0.78 & $1.45^{\star *}$ & 0.56 & 0.67 \\
\hline little & chap & 1630 & 132 & 2 & 3.29 & 0.82 & $1.27^{\star \star}$ & 0.49 & 0.66 \\
\hline weary & night & 46 & 856 & 1 & 4.74 & 0.87 & $0.96^{\star *}$ & 0.37 & 0.62 \\
\hline great & emotions & 911 & 45 & 1 & 4.68 & 0.87 & $0.96^{* *}$ & 0.37 & 0.62 \\
\hline things & behind & 622 & 415 & 2 & 3.03 & 0.81 & $1.24^{* *}$ & 0.48 & 0.65 \\
\hline very & dark & 1410 & 279 & 2 & 2.42 & 0.76 & $1.15^{\star \star}$ & 0.45 & 0.61 \\
\hline right & about & 757 & 1723 & 4 & 1.69 & 0.68 & $1.38^{* *}$ & 0.54 & 0.61 \\
\hline *make & sense & 963 & 192 & 1 & 2.51 & 0.77 & $0.82^{* *}$ & 0.32 & 0.55 \\
\hline only & chance & 1187 & 200 & 1 & 2.15 & 0.74 & $0.77^{* *}$ & 0.30 & 0.52 \\
\hline little & after & 1630 & 1304 & 4 & 0.98 & 0.52 & $0.99^{* *}$ & 0.38 & 0.45 \\
\hline
\end{tabular}

*actual collocation $\quad * *$ Rejected for collocation $(|t|<2.57)$ 
Table 3: Precision and recall for Mutual Information.

\begin{tabular}{lcc}
\hline $\begin{array}{c}\text { Mutual Information } \\
\text { (equal to or more than) }\end{array}$ & $\begin{array}{c}\text { Precision } \\
(\text { in } \%)\end{array}$ & $\begin{array}{c}\text { Recall } \\
\text { ( in \%) }\end{array}$ \\
\hline 10.0 & 43 & 18 \\
8.0 & 35 & 35 \\
6.0 & 39 & 82 \\
4.0 & 27 & 88 \\
\hline
\end{tabular}

Table 4: Precision and recall for fuzzy decision model

\begin{tabular}{ccc}
\hline $\begin{array}{c}\text { Grade of membership } \\
\text { ( equal to or more than) }\end{array}$ & $\begin{array}{c}\text { Precision } \\
\text { (in \%) }\end{array}$ & $\begin{array}{c}\text { Recall } \\
\text { ( in \%) }\end{array}$ \\
\hline 0.98 & 100 & 12 \\
$\mathbf{0 . 9 5}$ & $\mathbf{7 9}$ & $\mathbf{6 5}$ \\
0.90 & 48 & 76 \\
0.85 & 38 & 76 \\
\hline
\end{tabular}

From table 3 and 4, we can observe that fuzzy set theoretical model based on mutual information and t-score provides a better opportunity to extract collocations than using mutual information and t-score alone. Particularly, at more than or equal to .95 grade of membership the model has shown a good result.

\section{DISCUSSION}

The present work was carried out to utilize the fuzzy approach for collocation extraction. We have calculated the mutual information scores and t-scores for different word combinations. We have found that these two methods alone are not sufficient to extract collocations; however these methods form a strong basis for collocation extraction. In mutual information score it is tough to decide which score can be considered as high score and in t-score the values less than but near to the chosen value of ' $t$ ' may have the capability of making collocations. These points have opened the way to think in the direction of utilizing the fuzzy set theoretic approach and we have fuzzified both the techniques. Results prove the utility of fuzzy approach for collocation extraction. Therefore we conclude that the proposed model based on fuzzy set theoretical approach opens a new dimension for collocation extraction.

\section{REFERENCES}

[1] Bellman, R.E., Zadeh, L. A. (1970). Decision making in fuzzy environment. Management Science 17(4) 141-164.
[2] Choueka, Y., Klien, T., Neuwitz, E. (1983). Automatic retrieval of frequent idiomatic and collocational expressions in a large corpus. Journal for Literary and Linguistic computing Vol 4, 34-38.

[3] Church Kenneth W., Hanks, Patrick. (1989). Word association norms, mutual information and lexicography. In Proceedings of the $27^{\text {th }}$ meeting of the Association of Computational Linguistics 76-83.

[4] Church, Kenneth W., Gale, William A. (1991). Concordance for parallel text. In proceedings of the seventh annual conference of the $U W$ centre for new $O E D$ and text research Oxford 40-62.

[5] Cambridge International Dictionary of Idioms (1998). UK, CUP.

[6] Dunning, Ted. (1993). Accurate methods for the statistics of surprise and coincidence. Computational Linguistics. Vol 19 61-74.

[7] Klir, George J. \& Yuan Bo. (2001). Fuzzy sets and fuzzy logic theory and application Prentice Hall of India

[8] Kathleen R. McKeown and Dragomir R. Radev . (2000). Online manual. Available at:

http://citeseer.ist.psu.edu/mckeown00collocations.html

[9] Lin, Dekang. (1998). Extracting collocations from text corpora. In first workshop on Computational terminology, Montreal, Canada.

[10] Manning, Christopher D., Schutze Heinrich. (2002). Foundations of Statistical Natural Language Processing, MIT Press.

[11] Smadja, Frank.(1993). Retrieving collocations from text: Xtract. Computational Linguistic Vol 19(1) 143177.

[12]Fazly, A. , Suzanne S. (2007). "Distinguishing Subtypes of Multiword Expressions Using Linguistically-Motivated Statistical Measures". In Proceedings of the Workshop on A Broader Perspective on Multiword Expressions, 9-16,

[13]Pecina Pavel. (2005). "An Extensive Empirical Study of Collocation Extraction Methods". In Proceedings of the ACL Student Research Workshop, 13-18,

[14] Seretan V. , Wehrli E. (2006). "Multilingual Collocation Extraction: Issues and Solutions". In Proceedings of the Workshop on Multilingual Language Resources and Interoperability, 40-49,

[15]Inkpen Diana Zaiu, Hirst Graeme. (2002). “Acquiring Collocations for Lexical Choice between Near-Synonyms" In Proceedings of the Workshop of the ACL Special Interest Group on the Lexicon. 67-76.

[16] Weeber Marc, Vos Rein. (2000). "Extracting the LowestFrequency Words: Pitfalls and Possibilities". Computational Linguistics Volume 26, Number 3, 301317. 